\title{
Necroptosis and Cancer
}

\author{
Ayaz Najafov ${ }^{1}$, Hongbo Chen ${ }^{1,2}$, and Junying Yuan ${ }^{1,}$, \\ ${ }^{1}$ Department of Cell Biology, Harvard Medical School, 240 Longwood Ave. Boston, MA 02115 \\ ${ }^{2}$ Department of Pediatrics, Union Hospital, Tongji Medical College, Huazhong University of \\ Science and Technology, Wuhan 430022, China
}

\begin{abstract}
Necroptosis is a programmed lytic cell death pathway, deregulation of which is linked to various inflammatory disorders. Escape from programmed cell death and inflammation play a significant role in cancer, and therefore, investigating the role of necroptosis in cancer has been of high interest. Necroptosis has been shown to promote cancer metastasis and T cells death. Escape from necroptosis via loss of RIPK3 expression is a feature of some cancers. While necroptosis is a promising novel target for cancer therapies, further investigation into its biological role in carcinogenesis is warranted. In this article, we review the recently-identified interplay points between necroptosis and cancer, and outline major biological questions that require further inquiry on the road to targeting this pathway in cancer.
\end{abstract}

\section{Keywords}

necroptosis; cancer; metastasis; cross-priming; therapy; immunity

\section{Necroptosis - a regulated necrotic cell death mechanism}

Necroptosis has been first discovered as a programmed cell death mode only a decade ago [1]. The importance of this pathway has driven an exponential interest in the cell death field and as a result the pathway has been extensively studied both on the basic and translational research fronts. While much remains to be learned about the mechanism and regulation of necroptosis, the solid recognition of the pathophysiological relevance of this pathway in human diseases has already led to clinical trials for developing small molecule drugs targeting necroptosis for the treatment of major diseases ranging from Crohn's disease and rheumatoid arthritis to amyotrophic lateral sclerosis and Alzheimer (Text Box 1). One of the major issues with cancer therapies is drug resistance due to escape from programmed cell death and therefore, it is important to address the question whether necroptosis plays a role in cancer resistance. Moreover, since excessive inflammation can promote cancer cell growth and metastasis, understanding the biological significance of a pro-inflammatory cell

\footnotetext{
*Corresponding author: jyuan@hms.harvard.edu.

Publisher's Disclaimer: This is a PDF file of an unedited manuscript that has been accepted for publication. As a service to our customers we are providing this early version of the manuscript. The manuscript will undergo copyediting, typesetting, and review of the resulting proof before it is published in its final citable form. Please note that during the production process errors may be discovered which could affect the content, and all legal disclaimers that apply to the journal pertain.
} 
death like necroptosis is critical for our understanding of cancer biology. Although much remains to be discovered and understood on this subject, with the advance of our understanding of the basic biology of this pathway, recent reports have started to address the role of necroptosis in cancers. These studies revealed that necroptosis in the tumor microenvironment may have been adapted by cancers to promote metastasis and thus, suggests the possibility of inhibiting necroptosis as an anti-metastasis strategy. On the other hand, certain cancer cells may show a preferential reduction in the expression of key mediators of necroptosis, suggesting that necroptosis of cancer cells may negatively regulate tumorigenesis. In this article, we discuss the emerging roles of necroptosis in regulation of cancer cell growth and metastasis, as well as major unknowns in this field.

\section{Text Box 1}

\section{Necroptosis and inflammatory human diseases}

Necroptosis is involved in promoting inflammation under pathological conditions. Inflammation is one of the key cancer-fueling processes during tumorigenesis. Necroptosis has been implicated in several inflammatory neurodegenerative diseases, including amyotrophic lateral sclerosis (ALS) [46], multiple sclerosis [9] and NiemannPick disease [47]. A tight connection between necroptosis and intestinal inflammation has been established in various mouse models $[48,49]$. In children with inflammatory bowel disease (IBD), necroptosis was active and contributed to enhanced intestine inflammation [50]. Besides IBD, necroptosis plays a key role in the inflammatory skin disease [51,52]. A RIPK1 inhibitor has been advanced into in a Phase 1 human clinical trial for the treatment of AD and ALS by Denali Therapeutics Inc. RIPK1 inhibitor GSK2982772 developed by GlaxoSmithKline Pharmaceuticals is in Phase 2 clinical trials for the treatment of ulcerative colitis and psoriasis.

While the activation of caspases by ligands of the death receptor family, such as TNFa (Tumor Necrosis Factor alpha), FasL (Fas Ligand), and TRAIL (TNF-Related ApoptosisInducing Ligand), through binding to their cognate receptors, had been well-established to mediate the "extrinsic" apoptosis pathway [2], the unexpected discovery that inhibition of caspases under these conditions would turn apoptotic cell death into necrosis led to the investigation for the possible existence of a regulated necrotic cell pathway in mammalian cells by chemical biological approaches [1]. The activation of TNFR1 (Tumor Necrosis Factor Receptor 1) by TNFa leads to the formation of a transient intracellular multi-protein complex, called the TNF-R1 signaling complex - TNF-RSC (complex I) (Figure 1). TNFRSC provides a platform to orchestrate the activation of multiple signaling pathways by mediating a complex pattern of modifications such as ubiquitination and phosphorylation to collectively decide within minutes of TNFa stimulation if a cell may live or die. RIPK1 is recruited by the intracellular DD (death domain) motif of TNFR1, which also recruits TRADD (Tumor necrosis factor Receptor type 1 Associated Death Domain protein), an adaptor protein. TRADD in turn recruits FADD (Fas-Associating protein with Death Domain), a critical adaptor for the recruitment and activation of caspase-8, to promote apoptosis. TRADD also recruits TRAF2/TRAF5 (TNF Receptor-Associated Factor 2), redundant adaptors to mediate the recruitment of cIAP1/2 (cellular Inhibitor of Apoptosis 
Protein), two important E3 ubiquitin ligases that mediate the ubiquitination of RIPK1 [3].

Notably, key regulators of RIPK1 ubiquitination, namely Cyld and XIAP (X-linked Inhibitor of Apoptosis Protein) have been found to be mutated and overexpressed in cancer, respectively $[4,5]$ (further discussed below).

The ubiquitination status of TNF-RSC is critical in dictating multiple downstream events. The stimulation of TNFa may promote the activation of NF- $\mathrm{kB}$ (nuclear factor kappa B), a pro-inflammatory pathway that mediates cell survival and the production of multiple inflammatory cytokines. On the other hand, inhibition of NF- $\mathrm{kB}$ pathway output by protein synthesis inhibitor cycloheximide promotes the formation of complex-IIa which mediates the interaction of FADD and caspase-8 to promote apoptosis in RIPK1-independent manner. Alternatively, the treatment with Smac mimetics, the small molecules that can promote the activation of E3 ubiquitin ligase activity of cIAP1/2 to lead to its degradation via proteasome and that consequently reduces K63 ubiquitination of RIPK1, can lead to the activation of caspase- 8 and apoptosis in RIPK1-dependent manner, termed RIPK1-dependent apoptosis (RDA) [6,7].

Under apoptosis-deficient conditions, however, RIPK1 is activated, indicated by the phosphorylation of Ser166, an autophosphorylation marker for its activation [8-10]. The activated RIPK1 interacts with RIPK3 to form an alternative complex, termed complex IIb/ necrosome, where these kinases interact via their RIP homotypic interaction motif (RHIM) domains [11-13]. Activated complex-IIb/necrosome in turn mediates phosphorylation of MLKL (Mixed Lineage Kinase domain-Like protein), a pseudokinase, and the translocation of phosphorylated MLKL to plasma membrane to promote necrosis by disrupting the integrity of cell membrane [14-17].

While the necroptosis pathway mediated by TNFR1 upon stimulation of TNFa is best understood, the engagement of $\mathrm{T}$ cell receptors, interferon receptors, pattern recognition receptors (Toll-like receptors) and even genotoxic stimuli and anti-cancer drugs have been reported to activate necroptosis [18]. Since the production of TNFa may be a common consequence of different cellular stresses, the engagement of TNFR1 by TNFa might contribute to the activation of necroptosis in various cellular stress conditions. Given that escape from apoptosis is a hallmark of cancer, in certain contexts, such engagement will inevitably lead to tumor growth as a consequence of the NF- $\mathrm{kB}$ pathway activation or the pro-inflammatory effect of necroptotic cell death, suggesting that inhibition of necroptosis in cancer could have beneficial therapeutic effects via reduction of tumor growth-fueling inflammation levels in the tumor microenvironment.

\section{Necroptosis in the tumor microenvironment promotes inflammation and cancer metastasis}

Metastasis is the leading cause of cancer-related mortality. It is increasingly recognized that metastasis involves a complex interaction of cancer cells with their microenvironment including infiltrated immune cells and secreted cytokines, chemokines, and growth factors that collectively create an inflammatory milieu that promotes the invasive and metastatic ability of cancer cells. In this regard, as a proinflammatory form of cell death, necroptosis of 
non-transformed cells in the tumor microenvironment might facilitate metastasis by promoting inflammation. TNFa has been known to play a central role in tumor progression; however, the exact downstream mechanism of this progression has not been fully elucidated. Elevated expression of TNFa in the tumor microenvironment is a characteristic of many malignant tumors and is associated with poor prognosis [19]. Consistent with a proinflammatory nature of necroptosis and the pro-cancer role of inflammation, Nec-1 has been shown to reduce inflammation and colitis-associated tumorigenesis in a mouse model of the disease [20] (Text Box 2). Furthermore, Seifert et al. have shown that necroptosis promotes macrophage-induced suppression of $\mathrm{T}$ cell immunity in pancreatic ductal adenocarcinoma and consequently tumor cell metastasis, reinforcing the idea that targeting necroptosis in cancer can be a viable therapeutic strategy [21].

\section{Text Box 2}

\section{Defining a novel programmed cell death using chemical biology}

The discovery of the small molecule Nec-1 (methyl-thiohydantoin-Trp) and its improved analog Nec-1s (7-Cl-O-Nec-1) [5-(7-chloro-1H-indol-3-yl)methyl)-3-

methylimidazolidine-2,4-dione] as highly effective and specific inhibitors of necroptosis played an important role in defining this regulated necrotic cell death mechanism and in characterizing the involvement of necroptosis in various cell and animal models of human diseases $[1,8,18]$. The original screening hit Nec-1 has been noted to have an off-target effect on inhibiting indoleamine 2,3-dioxygenase (IDO) (IC50 117 $\mu \mathrm{M}$ ), which is about $58 \times$ higher than that of IC50 for Nec-1 to inhibit necroptosis $(2 \mu \mathrm{M})$; thus, the off-target effect of original Nec-1 in inhibiting IDO is not relevant when used at 10-20 $\mu \mathrm{M}[41,42]$. Consistently, 1-methyl-D, L-tryptophan (1-MT), an IDO inhibitor, cannot inhibit necroptosis even when used at very high concentration. The optimized Nec-1s has no activity towards IDO [41,42]. An oral formulation has been developed to deliver consistent amount of Nec-1s in animal models [9]. The requirement of RIPK1 in mediating caspase-independent death of Jurkat cells was first noted by Tschopp group [43]. The ability of Nec-1s to specifically inhibit the activity of RIPK1 (Receptorinteracting serine/threonine-protein kinase 1), without affecting its scaffolding function made it possible to determine the specific role of its kinase activity in a wide range of cultured systems and animal models, which was critical to convincingly demonstrate the existence of necroptosis as a common cell death mechanism, rather than some oddity of a specific cell line. In this regard, the chemical biology approach beautifully accomplished the goal of traditional genetic analysis in model organisms such as the nematode $\mathrm{C}$. elegans and fruit fly Drosophila, where point-mutations generated by random mutagenesis are required to painstakingly dissect functions mediated by different domains of multi-functional proteins. Nec-1s binds to a hydrophobic pocket between the $\mathrm{N}$ lobe and $\mathrm{C}$ lobe of the RIPK1 kinase domain and stabilizes the kinase in an inactive conformation by interacting with highly conserved amino acids in the activation loop and the surrounding structural elements [44]. Thus, Nec-1s is an excellent example of type IV kinase inhibitors which are known to show high selectivity towards their targets. Consistently, Nec-1s demonstrates exclusive selectivity towards RIPK1 [45]. Importantly, in addition to the upstream role of RIPK1 in the necroptosis pathway, the remarkable 
potency and selectivity of Nec-1s makes it an excellent tool for translational studies of the role of necroptosis in cancer.

Metastasis primarily involves the spreading of individual tumor cells through the circulatory system to colonize distant organs. The extravasation of tumor cells through endothelium is an important step in metastatic spread. Tumor cells were shown to induce necroptosis of endothelial cells by activating death receptor 6 (DR6 encoded by TNFRSF21), also a member of death receptor family, to promote tumor cell extravasation and metastasis [22]. Treatment of mice with Nec-1 or endothelial cell-specific deletion of RIPK3 or MLKL reduced tumor cell-induced endothelial necroptosis, tumor cell extravasation and metastasis. On the other hand, pharmacological caspase inhibition or endothelial cell-specific loss of caspase-8 promoted these processes. Thus, blocking necroptosis of endothelial cells might be a potential clinically-translatable antimetastatic therapeutic approach.

\section{Necroptosis and cancer immunity}

Cross-priming, the process that stimulates naïve cytotoxic $\mathrm{CD} 8^{+} \mathrm{T}$ cells, is necessary for immunity against most tumors [23]. Necroptotic cells were shown to initiate adaptive immunity by providing both antigens and inflammatory stimuli for dendritic cells (DCs), which in turn activate CD8+ T cells and anti-tumor immunity [24]. Using an artificial system that can promote the dimerization of RIPK3 upon treatment with a dimerizer, it was shown that the activation of RIPK3/RIPK1/NF- $\mathrm{kB}$ pathway can provide both antigen and immune stimulation, in turn, supporting DC-mediated cross-priming of CD8+ T cells. Cross-priming requires the scaffolding function of RIPK1 and nuclear factor- $\kappa \mathrm{B}$ (NF- $\kappa \mathrm{B})$ mediated transcription within the dying cells. In contrast, cells undergoing passive necrosis or secondary necrosis were poor inducers of $\mathrm{CD} 8^{+} \mathrm{T}$ cell responses in vivo. These observations suggest that a complex regulation of necroptosis mediators RIPK1 and RIPK3 in immune response towards tumors has to be carefully considered during cancer therapy designs involving necroptosis-targeting agents.

CD8+ T cells are key mediators of anti-cancer immunity. A subset of CD8+ T cells with $\mathrm{CD} 62 \mathrm{~L}^{\text {lo }}$ phenotype that are unable to survive for a long time in vivo may explain their inability to control tumor growth [25]. Therapeutically activating and maintaining the survival of tumor-infiltrating $\mathrm{T}$ lymphocytes are now regarded as critical in controlling the durability of cancer immune therapy [26]. In addition, in cancer immune therapy such as adoptive cell transfer therapy (ACT), which involves the engineering of T cells with tumor specific $\mathrm{T}$ cell receptors extracted from the patient, expanding them in vitro and then returning to the same patient to kill the cancer cells, a key limiting factor is the difficulty in obtaining sufficient quantity of $\mathrm{T}$ cells with tumor-specific $\mathrm{T}$ cell receptors. It has been shown that tumor reactive T cells might express elevated levels of RIPK1 and RIPK3 and undergo necroptosis upon T cell receptor (TCR) re-stimulation with cognate antigen, which can be inhibited by RIPK1 inhibitor [27]. In particular, the increased susceptibility of CD62L ${ }^{\text {lo }}$ to TCR re-stimulation induced necroptosis might be primarily responsible for decreased CD62 $\mathrm{L}^{\text {lo }} \mathrm{T}$ cell subset persistence. Inhibition of necroptosis of splenic $\mathrm{T}$ cells derived from Pmel mice, a mouse model that carries a rearranged $\mathrm{T}$ cell receptor transgene 
specific for the mouse homologue ( $\mathrm{pmel}^{S i}$ or pmel-17) of human premelanosome protein PMEL (also called gp100, and the T lymphocyte specific Thy $1^{a}$ (Thy1.1) allele and commonly used as a system for studies in immunotherapy, enhanced the survival of CD8+ T cells and reduced tumor burden. Thus, blocking necroptosis by inhibiting RIPK1 in activated tumor reactive T cells used in immunotherapy may improve the outcome of ACT as well as to promote the survival of tumor-infiltrating $\mathrm{T}$ cells to enhance the efficacy and application of cancer immune therapies.

\section{Down-regulation of key necroptosis mediators in cancer}

Down-regulated expression of multiple key mediators of necroptosis has been reported in cancers. CYLD is a deubiquitinating enzyme that promotes necroptosis [28]. Loss of the deubiquitinating activity of CYLD is involved in mediating familial cylindromatosis, an autosomal dominant predisposition to tumors of skin appendages called cylindromas. Downregulation of CYLD was also found in chronic lymphocytic leukemia, which led to resistance to TNFa-induced necroptosis [5]. The transcriptional repression by lymphoid enhancer-binding factor 1 (LEF1), a downstream effector of the Wnt/ $\beta$-catenin pathway, was involved in mediating down-regulation of CYLD. The transcription of CYLD was also found to be negatively regulated by Snail, which promotes tumor progression in malignant melanoma [29]. However, the consequence of down-regulating CYLD in cancers might not be limited to resistance of necroptosis per se, as CYLD also serves as a negative regulator of $\mathrm{NF}-\mathrm{kB}$, which is an important mediator of inflammation and tumor growth [30,31].

Reduced expression of RIPK3 has been reported in primary colorectal cancers, when compared with paired normal colorectal mucosa cells. In a cohort of 112 patients, low RIPK3 expression constituted an independent prognostic factor for overall survival and disease-free survival [32]. In addition, in an expression profiling study of RIPK1 and RIPK3 in CD34+ leukemia cells from a cohort of patients with acute myeloid leukemia and CD34+ cells from healthy donors, the expression of RIPK3, but not RIPK1, was significantly reduced in most cancer samples [33]. Moreover, down-regulated RIPK3 expression was found in malignant tissues from 75 breast carcinoma patients, regardless of tumor subtype [34]. Furthermore, the patients with greater than median RIPK3 mRNA expression exhibited improved metastatic relapse-free survival over a 10 -year period.

RIPK3 protein was found to be absent in about two-thirds of the $60+$ cancer cell lines tested [34]. While only $20 \%$ of examined hematopoetic cell lines lack RIPK3 expression, $80 \%$ of the other cell lines have no detectable RIPK3. The treatment with the hypomethylating agent decitabine restored RIPK3 expression in multiple cell lines, suggesting that the role of DNA methylation in silencing the expression of RIPK3 silencing. Consistently, knockdown of maintenance methyltransferase DNMT1 led to RIPK3 expression. Thus, reactivation of RIPK3 expression in cancer cells by modulators of DNA methylation might provide an opportunity for developing new anti-cancer treatments targeting necroptosis activation in cancer.

In addition, the expression of MLKL in cervical squamous carcinoma was found to be negatively correlated with histological grade, lymphatic metastasis, and low expression of 
MLKL indicated poor prognosis [35]. Similarly, reduced expression of MLKL in pancreatic adenocarcinoma and in resectable primary ovarian cancers was also found to be correlated with decreased overall survival and decreased disease-free survival $[36,37]$.

Taken together, down-regulating the expression of key mediators of necroptosis, including CYLD, RIPK3 and MLKL, appear to occur commonly at least in certain types of cancers. It is still unclear, however, as to the mechanisms that may have promoted such downregulation. Since necroptosis may be a common consequence of different cellular stress responses including chemotherapeutics, which may be directly or indirectly mediated by $\mathrm{TNFa}$, challenges for cancer cells to survive and grow in an intrinsically unhospitable environment may have provided selection pressure to select for low-expressers of CYLD, RIPK3 and MLKL. On the other hand, it is possible that the expression of dominant oncogenes may also provide selection pressure for cancer cells with resistance to necroptosis. In this regard, we still know very little.

RIPK3 may also mediate necroptosis-independent roles in cancers, such as inhibition of the inflammasome and regulation of malignant progenitor differentiation, which is inhibited in many acute myeloid leukemia (AML) patients [38]. RIPK3 knockout mice transplanted with bone marrow cells transduced with AML-driving mutant gene FLT3-ITD had a poorer survival than wild-type mice. Therefore, in certain cancers, targeting RIPK3 directly may lead to adverse effects.

\section{RIPK1-dependent apoptosis and cancers}

While the loss of CYLD, RIPK3, and MLKL expression may have the potential to increase the fitness of cancer cells, there is no evidence for a common loss of RIPK1 expression in cancers, which is not surprising as the loss of RIPK1 expression would result in sensitization to apoptosis and loss of cell fitness [39]. Thus, since most tumor cells maintain normal levels of RIPK1 expression, the activation of RIPK1-dependent apoptosis (RDA) might provide an opportunity for cancer treatment.

Overexpression of IAP family of proteins, including cIAP1, cIAP2 and XIAP, are often found in a variety of human tumors due to genomic amplification and has been linked to poor prognosis [4]. While it was originally thought that aberrant activation of IAPs might affect apoptosis in cancer cells by inhibiting caspases and promoting the pro-survival NF- $\mathrm{kB}$ signaling, it is now clear that a major role of cIAP1/2 is to mediate K63 ubiquitination of RIPK1. Down-regulation of cIAP1/2 by Smac mimetics promotes the activation of RIPK1, which in turn mediates RIPK1-dependent apoptosis under apoptosis-compatible conditions and necroptosis under apoptosis-deficient conditions. Thus, the retention of RIPK1 expression and down-regulation of key mediators of necroptosis in cancer cells suggests the possibility to activate RIPK1-dependent apoptosis as an anti-cancer therapeutic strategy [40].

\section{Concluding Remarks}

The importance of necroptosis in promoting metastasis and cross-priming, and its proinflammatory nature reinforce the notion of targeting it in cancer. However, 
downregulation of necroptosis mediators such as RIPK3 and MLKL in tumors suggests an escape mechanism from necroptosis in cancer (Figure 2). Therefore, in some cancers, induction of necroptosis to shrink tumors may require further therapeutic intervention to regain the expression of necroptosis mediators. Due to its essentiality for cellular fitness, RIPK1 expression, on the other hand, is not downregulated in cancer, opening doors to RDA-mediated cancer therapy development. Currently, there is not enough evidence for a conclusive global unilateral verdict about whether necroptosis promotes or restricts cancer cell growth and/or metastasis in general. Further research in various cancer types is required to determine whether induction versus inhibition of necroptosis/RDA has a significant therapeutic window. Moreover, important biological questions remain (see outstanding questions), such as (i) whether necroptosis contributes to carcinogenesis; (ii) how some cancers might lose RIPK3 expression and escape necroptosis; (iii) what might be the contribution of necroptosis to tumor microenvironment; (iv) how necroptosis may be manipulated to increase the efficacy of cancer immune therapies.

\section{Outstanding Questions Box}

- What is the contribution of necroptosis to the development of human cancers in general?

- What is the mechanism that promotes the loss of RIPK3 in certain cancers?

- What is the contribution of necroptosis to tumor microenvironment?

- Can necroptosis be modulated as a treatment for cancers?

- Can necroptosis of T cells be modulated to enhance cancer immune therapy?

\section{Glossary}

\section{Caspases}

A class of proteases activated by self-cleavage or cleavage by an upstream caspase that play role in various signaling events such as apoptosis, necroptosis and cytokine production by cleaving their target proteins. Inhibited by zVAD.fmk and Emricasan.

\section{Extrinsic apoptosis pathway}

caspase-dependent programmed cell death pathway initiated by binding of TNFa, FasL or TRAIL ligands with their cognate receptors TNFR1, Fas and TRAIL-R1

\section{MLKL}

Mixed lineage kinase domain-like protein. A pseudokinase that binds to membranal phospholipids upon phosphorylation downstream of RIPK3 activation. Upon oligomerization, forms a channel-like structure that promotes cell membrane disruption. Inhibited by compounds necrosulfonamide and GW806742X.

\section{Necroptosis}

Lytic, caspase-independent, programmed cell death pathway mediated by the RIPK1/RIPK3/ MLKL signaling axis and dependent on the kinase activity and oligomerization of the 
RIPK1 and RIPK3 kinases, as well as oligomerization and membranal translocation of MLKL pseudokinase. This pro-inflammatory pathway has been linked to cancer and metastasis.

\section{RDA}

RIPK1-dependent apoptosis pathway. Programmed cell death pathway that depends on caspase- 8 and RIPK1 kinase activity. This pathway, unlike canonical apoptosis, can therefore be inhibited by inhibitors of RIPK1 such as Nec-1.

\section{RIPK1}

Receptor-interacting serine/threonine-protein kinase 1. Central kinase for cellular decision point for initiation of either apoptosis, necroptosis or NF- $\kappa B$ pathway activation. Kinase activity is important for necroptosis and RIPK1-dependent apoptosis, but not NF- $\mathrm{KB}$ pathway activation, where RIPK1 serves as a scaffold to recruit additional signaling players required for the NF- $\mathrm{kB}$ pathway activation. Inhibited by compounds Nec-1 and GSK2982772.

\section{RIPK3}

Receptor-interacting serine/threonine-protein kinase 3. Promotes MLKL phosphorylation to initiate the oligomerization and membranal translocation of MLKL. Has been implicated in mediation of apoptosis. Expression is frequently lost in various types of cancer. Inhibited by compounds GSK'840, GSK'843 and GSK'872.

\section{TNFa}

Tumor necrosis factor alpha. A cytokine that binds to the TNFR1/2 receptor. It is mainly secreted by macrophages. Can induce either cytokine production and cell proliferation, apoptosis, RIPK1-dependent apoptosis (RDA) or necroptosis, depending on the concurrent treatments and conditions. Gene symbol: TNF.

\section{TNFR1}

Tumor necrosis factor receptor 1. A single-pass type I transmembrane receptor (not a kinase) for the TNFa ligand that has docking sites for recruiting downstream signaling players such as TRADD and RIPK1 upon ligation of the receptor and its trimerization. Ligation of the receptor can induce either apoptosis, necroptosis or NF- $\mathrm{kB}$ pathways, depending on additional concurrent stimuli. Gene symbol: TNFRSF1

\section{Bibliography}

1. Degterev A, et al. Chemical inhibitor of nonapoptotic cell death with therapeutic potential for ischemic brain injury. Nat Chem Biol. 2005; 1:112-119. [PubMed: 16408008]

2. Degterev A, et al. A decade of caspases. Oncogene. 2003; 22:8543-8567. [PubMed: 14634618]

3. Pobezinskaya YL, et al. The function of TRADD in signaling through tumor necrosis factor receptor 1 and TRIF-dependent Toll-like receptors. Nat Immunol. 2008; 9:1047-1054. [PubMed: 18641653]

4. LaCasse EC, et al. IAP-targeted therapies for cancer. Oncogene. 2008; 27:6252-6275. [PubMed: 18931692]

5. Wu W, et al. Clinical significance of down-regulated cylindromatosis gene in chronic lymphocytic leukemia. Leuk Lymphoma. 2014; 55:588-594. [PubMed: 23725390] 
6. Wang L, et al. TNF-alpha induces two distinct caspase-8 activation pathways. Cell. 2008; 133:693703. [PubMed: 18485876]

7. Dondelinger Y, et al. NF- $x B$-Independent Role of IKKa/IKK $\beta$ in Preventing RIPK1 KinaseDependent Apoptotic and Necroptotic Cell Death during TNF Signaling. Mol Cell. 2015; 60:63-76. [PubMed: 26344099]

8. Degterev A, et al. Identification of RIP1 kinase as a specific cellular target of necrostatins. Nat Chem Biol. 2008; 4:313-321. [PubMed: 18408713]

9. Ofengeim D, et al. Activation of necroptosis in multiple sclerosis. Cell Rep. 2015; 10:1836-1849. [PubMed: 25801023]

10. Berger SB, et al. Cutting Edge: RIP1 kinase activity is dispensable for normal development but is a key regulator of inflammation in SHARPIN-deficient mice. J Immunol. 2014; 192:5476-5480. [PubMed: 24821972]

11. Zhang DW, et al. RIP3, an energy metabolism regulator that switches TNF-induced cell death from apoptosis to necrosis. Science. 2009; 325:332-336. [PubMed: 19498109]

12. Cho YS, et al. Phosphorylation-driven assembly of the RIP1-RIP3 complex regulates programmed necrosis and virus-induced inflammation. Cell. 2009; 137:1112-1123. [PubMed: 19524513]

13. He S, et al. Receptor interacting protein kinase-3 determines cellular necrotic response to TNFalpha. Cell. 2009; 137:1100-1111. [PubMed: 19524512]

14. Sun L, et al. Mixed lineage kinase domain-like protein mediates necrosis signaling downstream of RIP3 kinase. Cell. 2012; 148:213-227. [PubMed: 22265413]

15. Chen $X$, et al. Translocation of mixed lineage kinase domain-like protein to plasma membrane leads to necrotic cell death. Cell Res. 2014; 24:105-121. [PubMed: 24366341]

16. Cai Z, et al. Plasma membrane translocation of trimerized MLKL protein is required for TNFinduced necroptosis. Nat Cell Biol. 2014; 16:55-65. [PubMed: 24316671]

17. Murphy JM, et al. The pseudokinase MLKL mediates necroptosis via a molecular switch mechanism. Immunity. 2013; 39:443-453. [PubMed: 24012422]

18. Zhou W, Yuan J. SnapShot: Necroptosis. Cell. 2014; 158:464-464e1. [PubMed: 25036639]

19. Wu Y, Zhou BP. Inflammation: a driving force speeds cancer metastasis. Cell Cycle. 2009; 8:32673273. [PubMed: 19770594]

20. Liu ZY, et al. Necrostatin-1 reduces intestinal inflammation and colitis-associated tumorigenesis in mice. Am J Cancer Res. 2015; 5:3174-3185. [PubMed: 26693068]

21. Seifert $\mathrm{L}$, et al. The necrosome promotes pancreatic oncogenesis via CXCL1 and Mincle-induced immune suppression. Nature. 2016; 532:245-249. [PubMed: 27049944]

22. Strilic B, et al. Tumour-cell-induced endothelial cell necroptosis via death receptor 6 promotes metastasis. Nature. 2016; 536:215-218. [PubMed: 27487218]

23. Bevan MJ. Cross-priming. Nat Immunol. 2006; 7:363-365. [PubMed: 16550200]

24. Yatim N, et al. RIPK1 and NF- $\kappa$ B signaling in dying cells determines cross-priming of CD8 ${ }^{+} \mathrm{T}$ cells. Science. 2015; 350:328-334. [PubMed: 26405229]

25. Klebanoff CA, et al. Central memory self/tumor-reactive CD8+ T cells confer superior antitumor immunity compared with effector memory T cells. Proc Natl Acad Sci U S A. 2005; 102:95719576. [PubMed: 15980149]

26. Sharma P, Allison JP. The future of immune checkpoint therapy. Science. 2015; 348:56-61. [PubMed: 25838373]

27. Kesarwani $\mathrm{P}$, et al. Blocking TCR restimulation induced necroptosis in adoptively transferred $\mathrm{T}$ cells improves tumor control. Oncotarget. 2016; 7:69371-69383. [PubMed: 27750220]

28. Hitomi J, et al. Identification of a molecular signaling network that regulates a cellular necrotic cell death pathway. Cell. 2008; 135:1311-1323. [PubMed: 19109899]

29. Massoumi R, et al. Down-regulation of CYLD expression by Snail promotes tumor progression in malignant melanoma. J Exp Med. 2009; 206:221-232. [PubMed: 19124656]

30. Trompouki E, et al. CYLD is a deubiquitinating enzyme that negatively regulates NF-kappaB activation by TNFR family members. Nature. 2003; 424:793-796. [PubMed: 12917689]

31. Brummelkamp TR, et al. Loss of the cylindromatosis tumour suppressor inhibits apoptosis by activating NF-kappaB. Nature. 2003; 424:797-801. [PubMed: 12917690] 
32. Feng X, et al. Receptor-interacting protein kinase 3 is a predictor of survival and plays a tumor suppressive role in colorectal cancer. Neoplasma. 2015; 62:592-601. [PubMed: 25997957]

33. Nugues AL, et al. RIP3 is downregulated in human myeloid leukemia cells and modulates apoptosis and caspase-mediated p65/RelA cleavage. Cell Death Dis. 2014; 5:e1384. [PubMed: 25144719]

34. Koo GB, et al. Methylation-dependent loss of RIP3 expression in cancer represses programmed necrosis in response to chemotherapeutics. Cell Res. 2015; 25:707-725. [PubMed: 25952668]

35. Ruan J, et al. Mixed lineage kinase domain-like protein is a prognostic biomarker for cervical squamous cell cancer. Int J Clin Exp Pathol. 2015; 8:15035-15038. [PubMed: 26823841]

36. He L, et al. Low expression of mixed lineage kinase domain-like protein is associated with poor prognosis in ovarian cancer patients. Onco Targets Ther. 2013; 6:1539-1543. [PubMed: 24204164]

37. Colbert LE, et al. Pronecrotic mixed lineage kinase domain-like protein expression is a prognostic biomarker in patients with early-stage resected pancreatic adenocarcinoma. Cancer. 2013; 119:3148-3155. [PubMed: 23720157]

38. Höckendorf U, et al. RIPK3 Restricts Myeloid Leukemogenesis by Promoting Cell Death and Differentiation of Leukemia Initiating Cells. Cancer Cell. 2016; 30:75-91. [PubMed: 27411587]

39. Ofengeim D, Yuan J. Regulation of RIP1 kinase signalling at the crossroads of inflammation and cell death. Nat Rev Mol Cell Biol. 2013; 14:727-736. [PubMed: 24129419]

40. Brumatti G, et al. The caspase- 8 inhibitor emricasan combines with the SMAC mimetic birinapant to induce necroptosis and treat acute myeloid leukemia. Sci Transl Med. 2016; 8:339ra69.

41. Takahashi N, et al. Necrostatin-1 analogues: critical issues on the specificity, activity and in vivo use in experimental disease models. Cell Death Dis. 2012; 3:e437. [PubMed: 23190609]

42. Degterev A, et al. Activity and specificity of necrostatin-1, small-molecule inhibitor of RIP1 kinase. Cell Death Differ. 2013; 20:366. [PubMed: 23197295]

43. Holler N, et al. Fas triggers an alternative, caspase-8-independent cell death pathway using the kinase RIP as effector molecule. Nat Immunol. 2000; 1:489-495. [PubMed: 11101870]

44. Xie T, et al. Structural basis of RIP1 inhibition by necrostatins. Structure. 2013; 21:493-499. [PubMed: 23473668]

45. Christofferson DE, et al. A novel role for RIP1 kinase in mediating TNFa production. Cell Death Dis. 2012; 3:e320. [PubMed: 22695613]

46. Ito Y, et al. RIPK1 mediates axonal degeneration by promoting inflammation and necroptosis in ALS. Science. 2016; 353:603-608. [PubMed: 27493188]

47. Cougnoux A, et al. Necroptosis in Niemann-Pick disease, type $\mathrm{C} 1$ : a potential therapeutic target. Cell Death Dis. 2016; 7:e2147. [PubMed: 26986514]

48. Günther C, et al. Caspase-8 regulates TNF-a-induced epithelial necroptosis and terminal ileitis. Nature. 2011; 477:335-339. [PubMed: 21921917]

49. Welz PS, et al. FADD prevents RIP3-mediated epithelial cell necrosis and chronic intestinal inflammation. Nature. 2011; 477:330-334. [PubMed: 21804564]

50. Pierdomenico $\mathrm{M}$, et al. Necroptosis is active in children with inflammatory bowel disease and contributes to heighten intestinal inflammation. Am J Gastroenterol. 2014; 109:279-287. [PubMed: 24322838]

51. Bonnet MC, et al. The adaptor protein FADD protects epidermal keratinocytes from necroptosis in vivo and prevents skin inflammation. Immunity. 2011; 35:572-582. [PubMed: 22000287]

52. Dannappel M, et al. RIPK1 maintains epithelial homeostasis by inhibiting apoptosis and necroptosis. Nature. 2014; 513:90-94. [PubMed: 25132550] 
Trends Box: Interplay between necroptosis and cancer

- Some cancers escape necroptosis via loss of RIPK3 expression.

- Necroptosis promotes cell extravasation and cancer metastasis and is important for anti-tumor cell-based immunity

- $\quad$ Necroptosis is a promising novel target for cancer therapies. However, further investigation into the biological role of necroptosis pathway in carcinogenesis and cancer-specific role of this programmed cell death is warranted. 


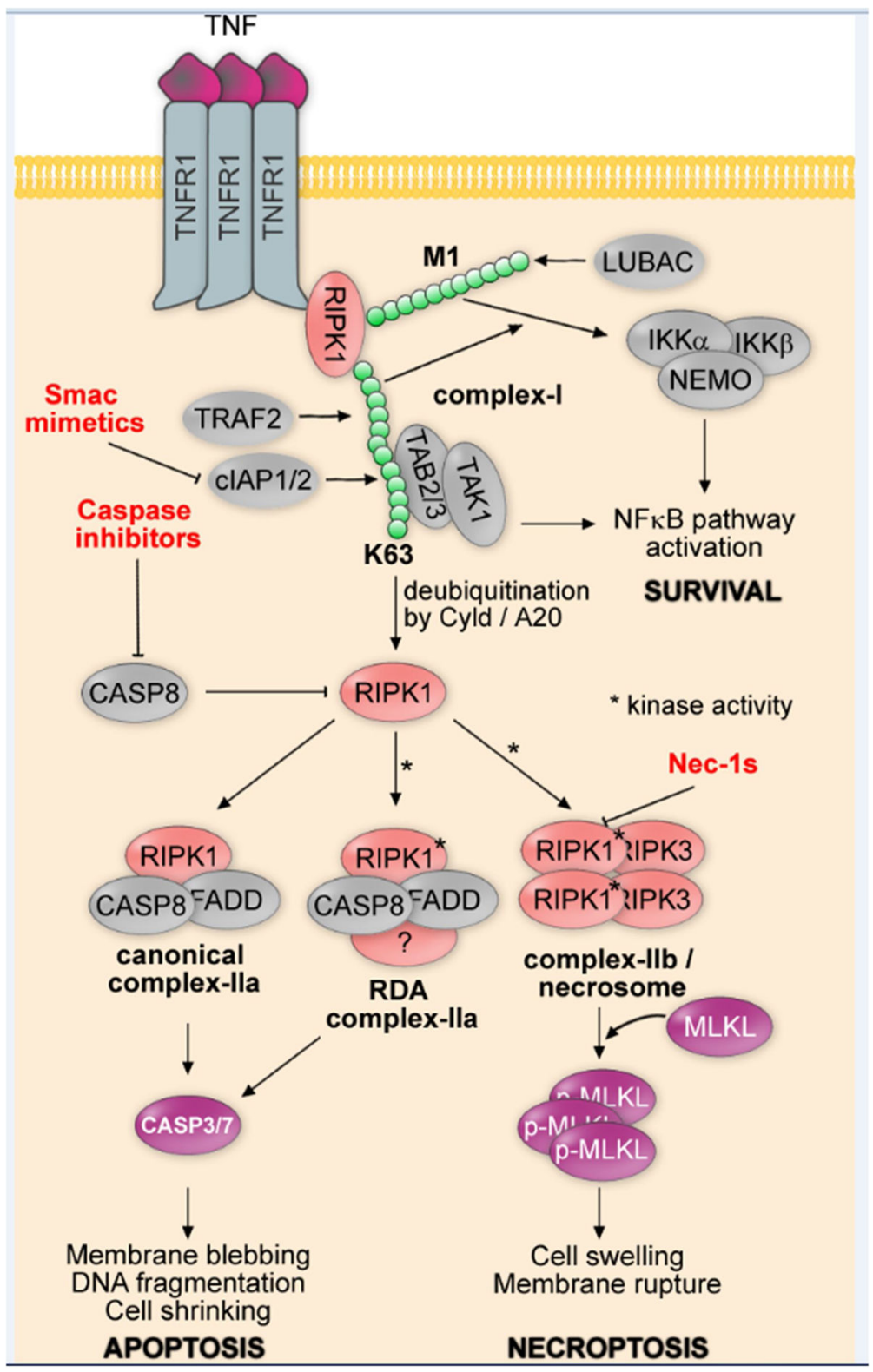

Figure 1. Canonical apoptosis, RIPK1-dependent apoptosis and necroptosis signaling cascades Tumor necrosis factor alpha (TNFa) ligation to tumor necrosis factor receptor 1 (TNFR1) results in receptor-interacting serine/threonine-protein kinase 1 (RIPK1) mediated formation of complex-I, which is mediated by E3 ubiquitin ligases cellular inhibitor of apoptosis protein (cIAP1/2) (and/or X-linked inhibitor of apoptosis protein XIAP) and TNF receptorassociated factor 2 (TRAF2), as well as linear ubiquitination complex (LUBAC). Complex-I promotes survival and cytokine production via I-kappa-B-kinase (IKK)- and transforming growth factor-beta-activated kinase 1 (TAK1)-mediated nuclear factor kappa B (NF- $\kappa \mathrm{B})$ pathway activation. Apoptosis, RIPK1-dependent apoptosis (RDA) or necroptosis pathways may be activated to promote cell death following deubiquitination of RIPK1 by Cyld and/or A20. While canonical complex-IIa does not require RIPK1 kinase activity, RDA complexIIa and complex-IIb/necrosome require RIPK1 kinase activity (*), which is inhibited by 
Nec-1s. Caspase8-caspase3 pathway is activated downstream of both canonical and RDA complex-IIa to promote apoptotic cell death, while Mixed lineage kinase domain-like protein (MLKL) phosphorylation and oligomerization downstream of complex-IIb/ necrosome to promotes necrotic cell death. 


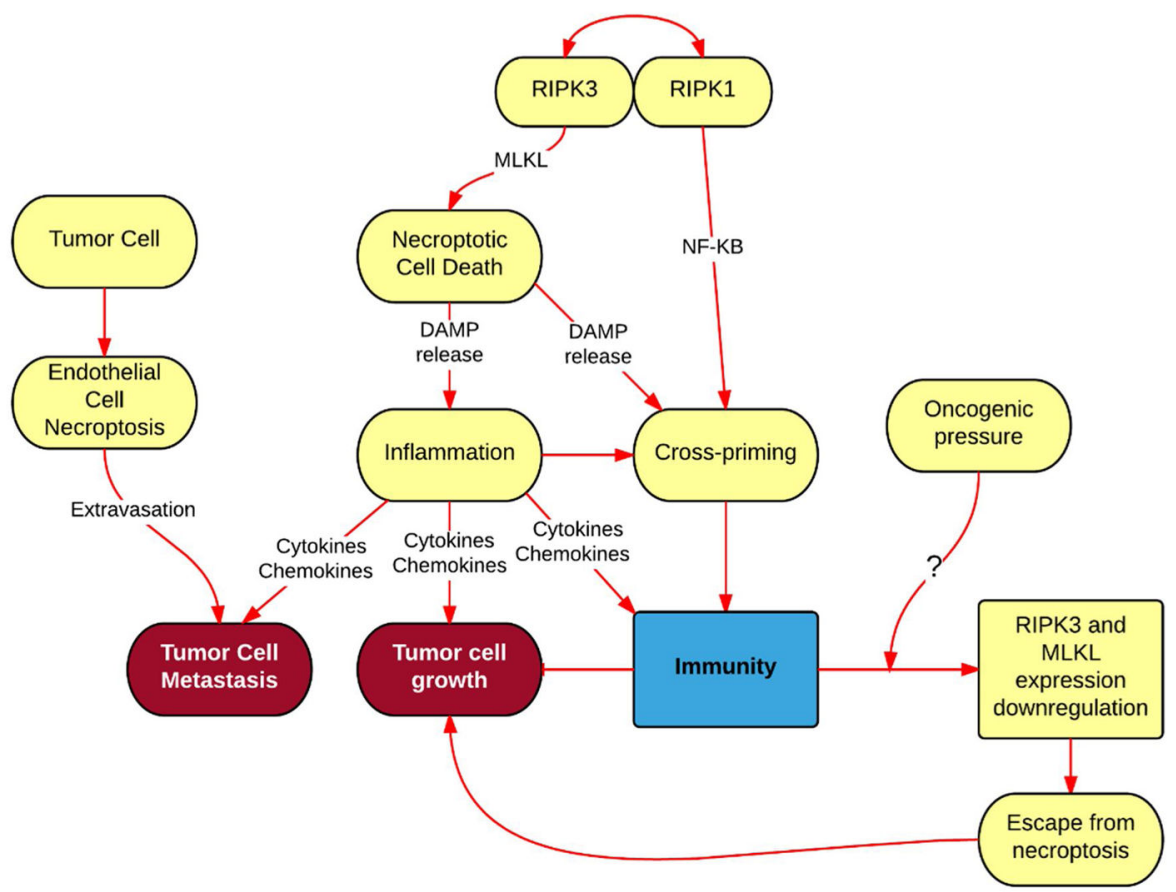

Figure 2. Role of necroptosis in cancer

Necroptotic cell death promotes inflammation, via release of DAMPS, fueling cancer growth and metastasis, while also promoting stimulation of naïve CD8+ T cells via an event called cross-priming, which is required for immune defense against cancer. Moreover, by killing endothelial cells via necroptosis, tumor cells achieve extravasation and metastasis, suggesting that inhibition of necroptosis in endothelial cells would restrict metastasis. While loss of RIPK3 an MLKL expression in many cancers results in escape from necroptosis, it is not know whether oncogenic forces play role in this process that results in escape from necroptosis. 